\title{
LIPID-BASED (LIPOSOMES, NANOSTRUCTURED LIPID CARRIERS) DELIVERY SYSTEMS FOR LOCAL ANESTHETICS
}

\author{
Eneida de Paula ${ }^{1 *}$ \\ ${ }^{1}$ Departamento de Bioquímica e Biologia Tecidual, \\ Universidade Estadual de Campinas (Unicamp), Campinas, SP, Brasil. \\ *depaula@unicamp.br
}

\section{RESUMO}

Local anesthetics (LA) are amphiphile molecules with the ability to block nerve condition, through the (reversible) binding to the voltage-gated sodium channel of excitable membranes. LA are broadly used to alleviate pain, in both medical and dentistry procedures. To extend the time of anesthesia and to decrease LA toxicity, different drug-delivery Systems (DDS) have been described. Among them, liposomes and nanostructured lipid carriers are interesting vehicles for drug delivery. Our group has developed different (conventional, elastic, ionic-gradient,...) liposomal formulations as well as solid lipid nanoparticles and nanostructured lipid carriers systems for the encapsulation of LA (dibucaine, lidocaine-prilocaine, ropivacaine, tetracaine) aiming infiltrative and topical anesthesia. We employed biophysical techniques (Magnetic Resonance, Dynamic Light Scattering, NanoTracking Analysis,...) to characterize the structural organization of the nanoparticles, and the LA interaction with the DDS. In vivo tests confirmed that encapsulation successfully prolonged the anesthesia time, and decreased the anesthetic doses and toxicity. Supported by FAPESP (\# 14/14457-5). 